\title{
Fault Identification Method of Ball Bearing Based on IAs and SVMs
}

\author{
Chao Chen, Zhaojun Yang,Heng Zhang, Binbin Xu ,Yifeng Ye and Yankun Wang \\ School of Mechanic Technology and Engineering, Jilin University, Changchun, 130025, China
}

\begin{abstract}
In order to effectively identify the bearing running condition, this paper proposed a new method which combines local mean decomposition (LMD) and support vector machine (SVM) together for ball bearing fault identification. Firstly, the gathered vibration signals were decomposed into a number of product functions (PFs) by LMD, with each PF corresponding to an instantaneous amplitude (IA) signal and instantaneous frequency (IF) signal. Then, introduce the concept of fault characteristic amplitude ratios which can be used to construct fault feature vectors; the extracted characteristic features were input into SVM to train and construct the fault identification model; the bearing running state identification was thereby realized. Cases of normal and fault were analyzed. Experimental results show that the proposed algorithm can diagnose the bearing failures reasonable and efficient.
\end{abstract}

\section{Introduction}

Bearing is the critical component of rotating machine which impacts the accuracy, reliability and lifetime of the machine. What's more, it's a common failure unit in rotating machines. Once it has any sort of faults, it would make the machine break down and lead to serious economic lose. Therefore, it is important to recognize the bearing running state [1].

Vibration measurements and signal analysis is a commonly used fault-detection technique employed in condition monitoring of ball bearings [2].The majority of the rotating machines use ball or rolling element bearings. Each bearing has one inner-race, one out-race and a set of balls or rolling elements [3].The ball bearings produce characteristic vibrations [4,5]. And when a defect develops in ball bearings, vibration characteristics and levels change. The vibrations caused by faults with distinctive characteristics reveal important information about the defect development within them that can be measured and compared with reference ones in order to perform the fault detection and diagnosis [6]. Hence, examining the vibration spectrum shows the information on the fault pattern. Normally, the process of ball bearing fault identification includes data acquisition, feature extraction and pattern recognition, and the later two are the priority [7].

The vibration signal of bearing has two typical characters. The first one is that bearing defect characters always perform as nonlinear and non-stationary which would affect the separation of the target signals, especially weaken the feature extraction of the fault information greatly [8]. Secondly compared with the complex noise the bearing defect characters are very weak which are usually submerged in the noise signals and hard to be picked out [9].

Targeting the first character, local mean decomposition (LMD) is proposed and applied to the fault feature extraction of ball bearings as LMD is successful for nonlinear and non-stationary signals LMD is a self-adaptive signal processing method to demodulate amplitude and frequency modulated signals [10]. By using LMD, vibration signal can be decomposed into a number of product functions ( $\mathrm{PFs}$ ), each of which is the product of an envelope signal and a purely frequency modulated signal from which physically meaningful instantaneous frequencies (IF) and instantaneous amplitudes (IA) can be obtained. Since the decomposition of the LMD is based on the local characteristics time scale of the data, LMD can be applied to nonlinear and non-stationary process perfectly $[10,11]$.

As it is hard to obtain sufficient bearing fault samples in practice, in recent years, support vector machines (SVMs) have been widely applied for bearing fault diagnosis because SVMs specialize for small number of samples [12]. SVMs are supervised learning models with associated learning algorithms that analyze data, classify data and recognize fault patterns. Due to the noise in the vibration signal, the data need be preprocessing to get fault feature. Author uses Local mean decomposition (LMD) method to reduce the noise impact. And the fault feature vectors extracted by LMD were input into SVM to classify the fault pattern of ball bearing.

The rest of the article is organized as follows: a brief introduction to the LMD and SVMs are provided in Section 2 and 3 respectively. Section 4 presents the proposed method based on fault diagnosis method based on LMD and SVMs. Section 5 shows the detailed analysis of a case 
study using our proposed method. Section 6 draws a short conclusion.

\section{LMD}

LMD is a novel self-adaptive method based on partial characters of the signal which decomposes vibration signal into a linear combination of a set of mono-component signals. The LMD method is a fully data driven approach which involves progressively separating a FM component from an AM envelope signal and gets a series of PFs, instantaneous amplitude (IAs) and instantaneous frequency (IFs) [13]. The process of computing IA and IF by LMD is shown as Fig. 1.

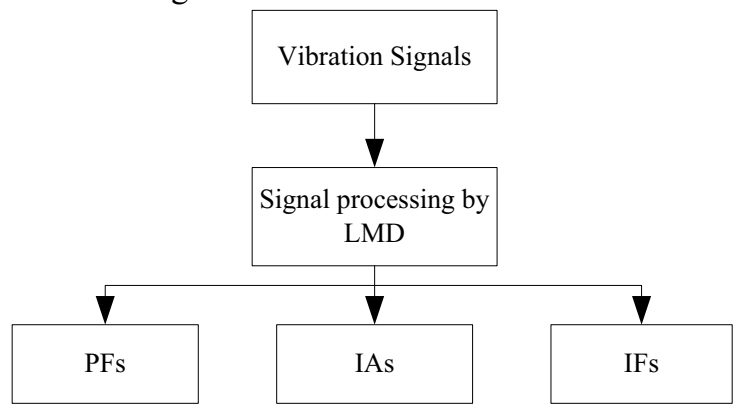

Figure 1. The sketch of computing IA and IF by LMD

\section{SVMs}

SVM is developed from the optimal separation plane under linearly separable condition. SVM uses a hypothesis space of linear functions in a high dimensional feature space to estimate decision surfaces directly rather than modeling a probability distribution across training data [14]. It uses support vector kernel to map the data from input space to a high dimensional feature space which facilitates the problem to be processed in linear form. The maximum-margin hyper-plane and margins are used to train trained samples from two classes ${ }^{15}$. The nearest samples are used to define the margin and are called support vectors. The basic principle of SVM is shown in the Fig. 2 .

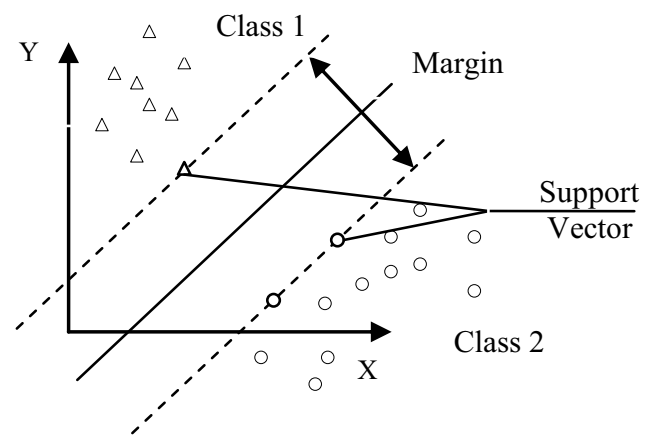

Figure 2. Basic principle of SVM

\section{Fault diagnosis dombined LMD and SVM}

While the ball bearing with different faults is operating, the corresponding special frequencies are produced in the vibration signals. Considering high-frequency band of vibration signal contains the key fault information of ball bearing [16], the fault characteristics could be extracted from the first numbers of PFs component. As PFs contain the information of the impulse and its severity level in each period, the fault characteristic parameters defined from PFs should reflect the working condition and fault pattern accurately. Namely, each PF obtained by LMD can be used to extract the fault feature of sample data. Yu [16] used Hilbert transformation to get the envelope spectrum of the envelope signal based on EMD. What's more, some characters of the envelope signal may be lost in Hilbert transformation. However, LMD gets the envelope spectrum directly and avoids information lost. Compared with support vector based on EMD, the support vector from LMD is more accuracy.

Based on the peak value could be found at corresponding fault characteristic frequencies in PFs when ball bearing has fault, three fault characteristics, namely fault instantaneous amplitude ratios (FIAR) $\gamma \quad \rho$ and $\eta$ are designated as:

$$
\gamma=\frac{A\left(f_{o}\right)}{A\left(f_{i}\right)}, \rho=\frac{A\left(f_{r}\right)}{A\left(f_{i}\right)}, \eta=\frac{A\left(f_{c}\right)}{A\left(f_{i}\right)}
$$

where, $f_{i}, f_{o}, f_{b}$ are ball bearing inner-race, out-race and ball fault characteristic frequencies respectively. $A\left(f_{i}\right), A\left(f_{o}\right), A\left(f_{b}\right)$ and $A\left(f_{c}\right)$ are the corresponding instantaneous amplitude (IA) of the PFs at fault characteristic frequencies. The fault characteristic frequencies of the ball bearing with inner-race fault, out-race fault, ball fault and cage can be calculated as follows [17]:

$$
\begin{gathered}
f_{i}=\frac{1}{2}\left(1+\frac{d}{D_{m}} \cos \alpha\right) f_{n} Z \\
f_{o}=\frac{1}{2}\left(1-\frac{d}{D_{m}} \cos \alpha\right) f_{n} Z \\
f_{b}=\frac{1}{2} \frac{D_{m}}{d}\left(1-\left(\frac{d}{D_{m}}\right)^{2} \cos ^{2} \alpha\right) f_{n} Z \\
f_{c}=\frac{1}{2}\left(1-\frac{d}{D_{m}} \cos \alpha\right) f_{n}
\end{gathered}
$$

where $d$ and $Z$ are the diameter and number of the rolling elements, respectively; $D_{m}$ is the pitch circle diameter; $\alpha$ is the contact angle of the roller bearing; $f_{n}$ is the rotation frequency of the shaft.

The fault diagnosis method is given as following:

1)Sample $N$ times at a certain frequency $f_{s}$ under the condition that the ball bearing is normal, the ball bearing with inner-race faults, the ball bearing with out-race faults and the ball bearing with ball faults, respectively. And the $4 \mathrm{~N}$ signals are taken as samples that are divided into two subsets, the training samples and test samples. 
2) The original vibration signals are decomposed into some PFs and the first numbers of PFs that include the most dominant fault information are chosen to extract the feature.

3) For each PF, get the corresponding IA and IF. According to Eq. (1) calculate the characteristic amplitude ratios $\gamma, \rho$ and $\eta$.

4) Design SVM classifiers. For SVM1, define the condition with inner-race fault as $\mathrm{y}=1$ and the other 3 conditions as $y=-1$ thus the inner-race fault could be separated from other conditions by SVM1. Then, define the condition with outer-race fault as $\mathrm{y}=1$ and the other conditions as $y=-1$ for SVM2, thus the outer-race fault could be separated from normal and ball fault condition by SVM2. Similarly, the ball fault could be separated from normal condition by SVM3.

5) The characteristic amplitude ratios of the training samples serve as the fault feature vectors to be input to the SVM and the classifiers are trained.

6) Actually the decision function $f(x)$ is determined only by the support vectors, so after they are obtained the fault characteristic vectors of test samples can be input to the trained SVM classifiers and then the working condition can be identified by the output of the SVM classifiers.

The flow chart of the identification method based on LMD and SVM are show in Fig. 3

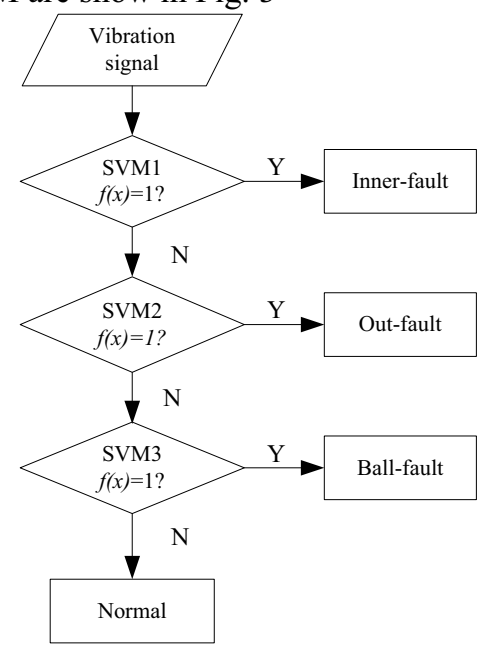

Figure 3. The flow chart of fault identification by SVM1, SVM2 and SVM3

\section{Data acquisition and signal preprocessing}

To evaluate the effectiveness of the proposed method based on LMD and SVMs for bearings, the test data were acquired by bearing accelerometer sensors under different operating loads and bearing conditions. The test bearing type is $7205 \mathrm{C}$, which is a deep groove ball bearing. Accelerometer sensors were placed at the 12 o'clock position at the drive end of the motor housing. The test bearings support the motor shaft and the load was at the speed of $1800 \mathrm{rpm}$. The sampling frequency is $10 \mathrm{kHz}$; and the sampling points are 1000 . The vibration signals of ball bearing were selected as normal, out-race fault, inner-race fault and ball fault.

Eight vibration signals of ball bearings in each condition are obtained at random in which 2 groups are drawn out respectively as the training samples and the rest are taken as test data. Decompose the vibration signals of above four conditions, namely normal, inner-race fault, out-race fault and ball fault with LMD method into a number of PFs according to Ref. [13]. The one group results of LMD decomposition are shown in Fig. 4, respectively. The decomposed results have $3 \mathrm{PFs}$ and 1 residue. The residue can be decomposed furthermore, but it makes no sense.

As main information of fault vibration signal of ball bearing is included in high-frequency bands, only first three PFs are chosen. According to Eqs. (2)-(5), the calculating results of fault characteristic frequencies of the ball bearing with inner-race fault, out-race fault ball fault and cage are $107.36 \mathrm{~Hz}, 162.19 \mathrm{~Hz}, 141.17 \mathrm{~Hz}$ and $18.02 \mathrm{~Hz}$, respectively. According to Eqs. (1), calculate the characteristic amplitude ratios $\gamma_{i}, \rho_{i}$ and $\eta_{i}$ in each PF envelope spectrum, which denotes the characteristic amplitude ratios of PF1, PF2 and PF3.

Table 1. The identification results of proposed method for ball bearing

\begin{tabular}{|c|c|c|c|c|c|c|c|c|c|c|}
\hline \multirow{2}{*}{ Test samples } & \multicolumn{3}{|c|}{ Characteristic vector } & \multicolumn{2}{c|}{ SVM1 } & \multicolumn{2}{c|}{ SVM2 } & \multicolumn{2}{c|}{ SVM3 } & \multirow{2}{*}{ Fault pattern } \\
\cline { 2 - 11 } & $\mathbf{( 1 )}$ & $\mathbf{( 2 )}$ & $\mathbf{( 3 )}$ & Distance & Re. & Distance & Re. & Distance & Re. & \\
\hline Inner-race fault & 0.7121 & 0.5524 & 0.4486 & 0.9120 & 1 & & & & & Inner-race fault \\
\hline Inner-race fault & 0.5141 & 0.5554 & 0.6129 & 1.0531 & 1 & & & & & Inner-race fault \\
\hline Out-race fault & 15.9789 & 28.0421 & 17.1438 & -22.8354 & -1 & 38.6380 & 1 & & & Out-race fault \\
\hline Out-race fault & 4.5184 & 7.6925 & 6.6721 & -4.3762 & -1 & 9.2407 & 1 & & & Out-race fault \\
\hline Ball fault & 1.3546 & 3.7431 & 2.3479 & -2.0211 & -1 & -4.2254 & -1 & 5.3312 & 1 & Ball fault \\
\hline Ball fault & 1.3650 & 3.7159 & 1.8772 & -1.8644 & -1 & -3.9307 & -1 & 6.0255 & 1 & Ball fault \\
\hline Normal & 0.6381 & 1.2295 & 1.7870 & -0.9537 & -1 & -1.3854 & -1 & -2.7208 & -1 & Normal \\
\hline Normal & 0.7762 & 0.8196 & 2.0920 & -0.7074 & -1 & -0.9113 & -1 & -1.3113 & -1 & Normal \\
\hline
\end{tabular}


Serve $\gamma_{i}$ and $\rho_{i}$ as the fault characteristic vectors to input to the SVM classifiers. By Ref. [14], design theclassifiers SVM1-3. After SVM classifiers have been trained, the fault characteristic vectors of test samples are input into the trained SVM classifiers and then the working condition is classified by the output of the SVM classifiers.
In this progress, the inner-race fault is indentified by SVM1successfully. Finally, by SVM1-3, all the classification results are shown in Table 1 from which we can see that three SVM classifiers can identify the working conditions and fault patterns of ball bearing accurately.
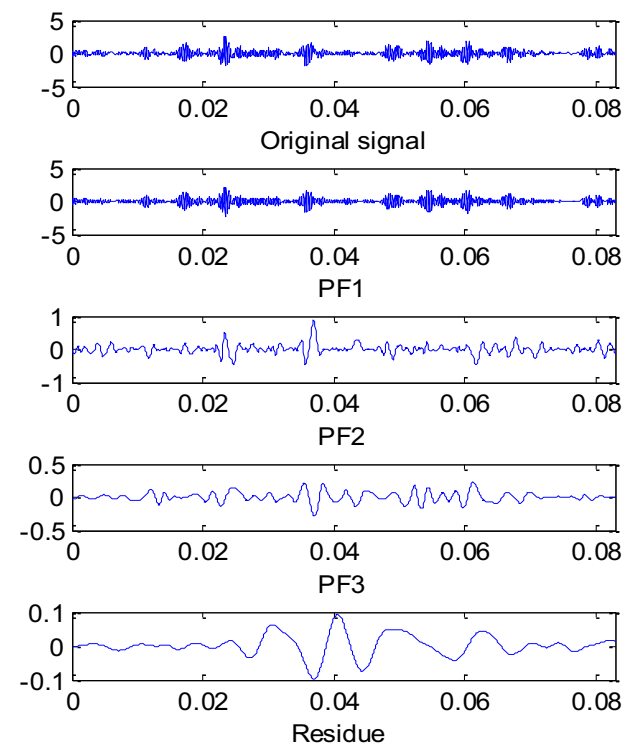

(b). Ball bearing with inner-race fault

(a). Normal ball bearing
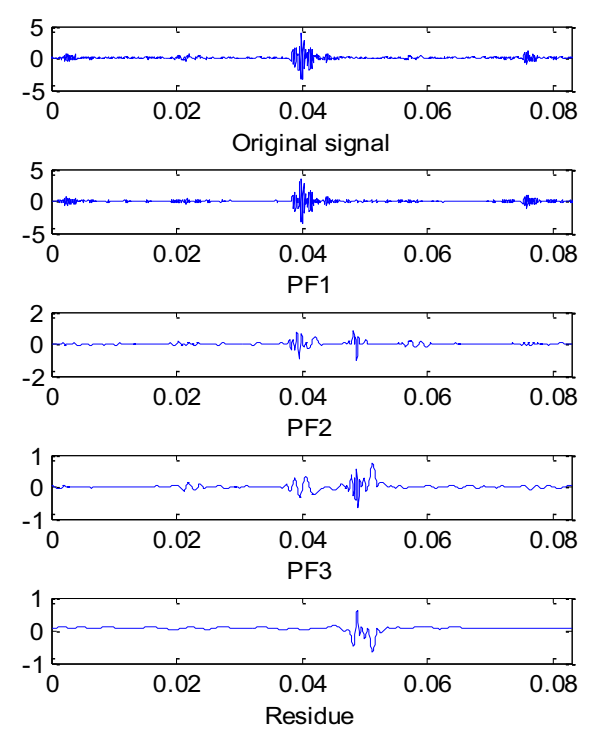

(c). Ball bearing with out-race fault
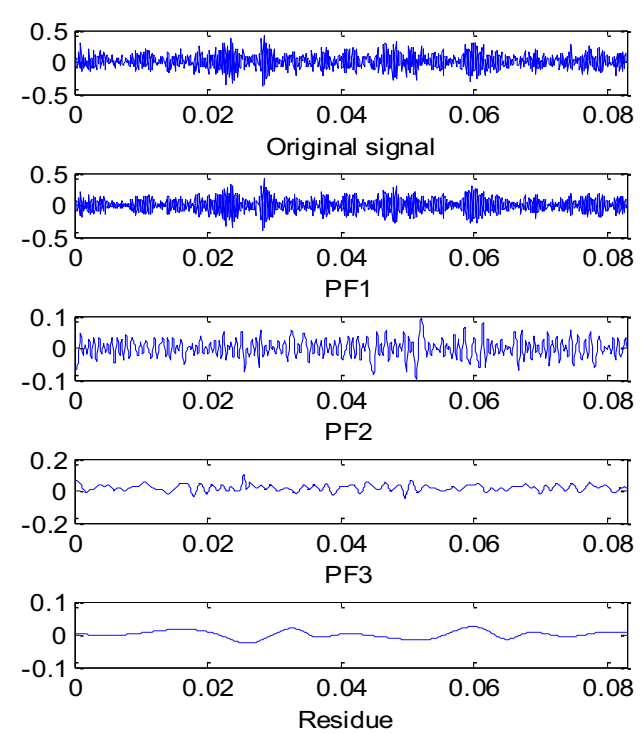

(d). Ball bearing with ball fault

Figure 4. The original signal and LMD decomposed results of ball bearing

\section{Conclusions}

In this paper, original vibration signals of ball bearing are decomposed by LMD. Considering each PF corresponding to an IA signal and IF signal could reflect the intrinsic and real information of the analyzed signal, the characteristic amplitude ratios of fault characteristic frequencies in fault bearing and corresponding frequencies of normal one in each IA are proposed. And the fault characteristic extraction method based on PF envelope spectrum is proposed in this paper. In addition, unlike EMD needs Hilbert transform to get the IAs and IFs, SVM which gets 
the two signals directly avoids some information losing. It's more accuracy than EMD method. The paper shows the analysis results of four kinds of ball bearing vibration signals among which one is normal and the other three have inner-race, out-race and ball faults, respectively, that the fault diagnosis approach of ball bearing based on PF, IA and SVM can be applied to classify the working conditions and fault patterns of ball bearing effectively and accurately.

\section{Acknowledgments}

This work was financially supported by the Specialized Research Fund of the Application engineering of one thousand sets of CNC machines with Dometic CNC systems (Grant No. 2014ZX040015061), the Postdoctoral fund project of Jilin province (Grant No. 801130020414)

\section{References}

1. W. Guo, P.W. Tse. A, Djordjevich, Faulty bearing signal recovery from large noise using a hybrid method based on spectral kurtosis and ensemble empirical mode decomposition, Meas. 45 (2012) 1308-1322

2. M. Zhao, J. Lin, X. Q. Xu, X. J. Li, Multi-fault detection of rolling element bearings under harsh working condition using IMF-based adaptive envelope order analysis, Sen 14(2014) 20320-20346

3. W. T. Sui, S. Osman, W. Wang, An adaptive envelope spectrum technique for bearing fault detection, Meas. Sci. Tech, 25(2014) 095004

4. J.P. Yang, S.X. Chen, Vibration predictions and verifications of disk drive spindle system with ball bearings. Comput Str. 80(2002)1409-18.

5. R. Aini, H. Rahnejat, R.Gohar, Vibration modeling of rotating spindles supported by lubricated bearings. J Tri.124( 2002)158-65.

6. P. Konar, P. Chattopadhyay. Bearing fault detection of induction motor using wavelet and Support VectorMachines (SVMs). Appl. Soft Com 11 (2011) 4203-4211

7. J. Yang, C.K. Peng, Y.S. Xu. Hierarchical entropy analysis for biological signals, J mput. Appl. Math 236 (2011) 728-742.

8. H. Xu, G. Chen. An intelligent fault identification method of rolling bearings based on LSSVM optimized by improved PSO. Mech. Sys. Sig. Pro 35 (2013) 167-175

9. B.T.Holm-Hansen, R.X. Gao, Vibration Analysis of a Sensor Integrated Ball Bearing. J Vib. Acou 122 (2000) 384-392

10. J. S. Smith, The local mean decomposition and its application to EEG perception data, J. R. Soc. Int 2 (2005) 443-454.

11. Y, Bu, J, Wu, J, Ma, et. al. The rolling bearing fault diagnosis based on LMD and LS-SVM. $26^{\text {th }}$ CCDC 3797-3801
12. N. Cristianini, J. Shawe-Taylor. Support Vector Machines and other Kernel Based Learning Methods, Cambridge University Press, 2000

13. Y. Wang, Z. He, Y. Zi, A demodulation method based on improved local mean decomposition and its application in rub-impact fault diagnosis. Meas. Sci. Tech. 20(2009)025704

14. J. Chenga, D. Yua, J.Tangb, Y.Yanga, Application of SVM and SVD technique based on EMD to the fault diagnosis of the rotating machinery. Shoc. Vib. 16(2009) 89-98

15. J. Lee. Advanced Electrical and Electronics Engineering, Springer Science \& Business Media, 2(2011) 425

16. Y. Yang, D. Yu, J. Cheng. A fault diagnosis approach for roller bearing based on IMF envelope spectrum and SVM. Meas. 40 (2007) 943-950

17. S. Nandi, H. A. Toliyat, X. D. Li, A. Richard, condition monitoring and fault diagnosis of electrical motors-a review, IEEE T Energy Conver, 20(2005) 719-729. 